\title{
Dairy consumption and risk of type-2 diabetes: the untold story
}

\author{
Sai Krishna Gudi \\ College of Pharmacy, Rady Faculty of \\ Health Sciences, University of Mani- \\ toba, Winnipeg, MB, Canada
}

The role of dairy products in human health has been extensively studied for decades; however, evidence regarding dairy consumption and risk of type 2 diabetes (T2D) remains controversial and uncertain. Furthermore, study results are misinterpreted to a remarkable extent. The aim of this review is to critically appraise the association between intake of dairy foods and risk of T2D. A thorough search was conducted using electronic databases of PubMed, Scopus, and Web of Science. Related studies that addressed this research question between 2004 to 2019 were considered. Although most of the existing evidence suggests a beneficial role of dairy consumption on risk of T2D, only low-fat dairy foods and yogurt have shown a significant and consistent role, while other dairy products showed no association with prevention of T2D. Researchers, readers, and the public should maintain caution when reporting and interpreting findings and consider aspects such as heterogeneity, generalizability, and clinical and statistical significance.

Keywords: Dairy products, Dairy consumption, Dairy foods, Type-2 diabetes, Metabolic syndrome, Insulin resistance

\section{Highlights}

It should be recognized that not all dairy products exhibit a beneficial role in preventing type-2 diabetes, except low-fat dairy foods, especially yogurt, while most other dairy foods have no association.

\section{Introduction}

Type 2 diabetes (T2D) affects approximately 463 million adults worldwide, a number that is estimated to reach 700 million by $2045{ }^{1)}$ T2D is a major public health concern, as 1 in 11 adults globally have T2D. ${ }^{2)}$ In addition to genetic and environmental factors, risk factors for T2D include advanced age, race/ethnicity, obesity, physical inactivity, high blood pressure, and sedentary lifestyle. ${ }^{3-5)}$ Thus, it is essential to identify modifiable risk factors causing T2D. Dairy products are important constituents of any diet, and the hormones present in dairy products, especially milk, are considered essential for growth and immunity. Dairy products such as milk, yogurt, cheese, and butter are considered essential for building healthy bones, teeth, gums, and maintaining a healthy weight because they are rich in calcium, vitamin D, vitamin A, magnesium, zinc, and protein. ${ }^{6}$ Calcium and vitamins that are present in dairy products assist with blood coagulation during injuries, protect nerves from damage, and help to build strong muscles. ${ }^{7)}$ However, evidence also suggests that consumption of dairy products promotes hormonal imbalances that may lead to several diseases, such as T2D, ${ }^{8}$ obesity, cardiometabolic syndrome, ${ }^{10)}$ and certain types of cancer. ${ }^{11)}$

In addition to their basic nutritional role, dairy products also contain a wide variety of hormones that mediate specific physiologic and pathologic functions of the endocrine
Address for correspondence: Sai Krishna Gudi

College of Pharmacy, Rady Faculty of Health Sciences, University of Manitoba, Winnipeg, MB R3E 0T5, Canada

Email:sknanu06@gmail.com,gudisk@ myumanitoba.ca

https://orcid.org/0000-0001-95769207
ISSN: 2287-1292(Online) 
system. ${ }^{12)}$ In the distant past, certain conflicts have been raised regarding dairy consumption as a contributing factor for developing T2D. Several prospective cohort studies, casecontrol studies, systematic reviews, and meta-analyses have assessed this association. The majority of their findings revealed an inverse relationship (i.e., protective effect) between dairy consumption and T2D risk. Although only a few studies conducted detailed analyses of a variety of dairy subgroups, the role and exact mechanisms of dairy products on metabolic diseases such as T2D remain unclear. Almost all studies have inferred a beneficial role of dairy products in preventing T2D; however, a remarkable number of studies showed either positive or no association of specific dairy foods with incidence of T2D. These results were not highlighted or prioritized in study conclusions. Some studies have underreported the harmful or nonassociation findings by not considering statistical significance, resulting in misinterpretation of results. Some results were not reflected in conclusions, and only the inverse association of dairy consumption was highlighted by ignoring other essential findings. Thus, this review places special emphasis on consumption of different dairy products and risk of T2D. The authors attempt to clarify this issue by drawing insight from many high-quality studies conducted around the world using real-world data. This will add to the current literature and assist the public in making informed decisions. A thorough search was conducted using electronic scientific databases of PubMed, Scopus, and Web of Science. Studies that addressed this research question between 2004 to 2019 were included in this review.

\section{Under- and overreporting and misinterpretation of findings}

\section{Evidence from epidemiologic studies}

In a study using data from the Health Professionals Follow-up Study, authors reported that risk of T2D decreased by $9 \%$ with an increase in total dairy intake by one serving per day. Thus, they concluded that dairy intake was associated with a modestly lower risk of T2D. However, multivariable relative risk (RR) for whole milk suggested that, compared to those who consumed whole milk less than once per month, there was a $19 \%$ higher risk of T2D for those who drank whole milk more than twice a week (RR, 1.19; 95\% confidence interval [CI], 1.0-1.43). This result was not reflected in their conclusions. ${ }^{13)}$ In a large prospective study, researchers inferred that an increase in dairy intake by one serving per day would lower the risk of T2D by $4 \%$. However, the confidence interval for RR crossed the level of no association (RR, 0.96; 95\% CI, 0.93-1.01). ${ }^{14)}$ In a Japan Public Health Center-based prospective study considering multivariable odds ratio with 95\% CI (OR, 0.71; 0.51-0.98), the authors reported that dairy food intake was significantly associated with a lower risk of T2D among women; however, the probability value suggests lack of significance at $P=0.054 .{ }^{15}$ )
Authors of the Blue Mountains Eye Study conducted in Australia concluded that there was no association between total dairy intake and risk of T2D, ignoring that people who had reduced/low-fat dairy foods exhibited $101 \%$ higher odds of metabolic syndrome/T2D, when comparing the highest quartile with the lowest quartile in a fully adjusted model (2.01, 95\% CI 1.05-3.83; $P=0.043){ }^{16)}$ In 2014, a nested case-control study conducted by O'Connor et al. ${ }^{17)}$ in the United Kingdom concluded that low-fat fermented dairy intake was associated with decreased risk of T2D. However, these implications were concluded by comparing only tertile 3 vs. tertile 1 (hazard ratio [HR], 0.76; 95\% CI, 0.60-0.99; $P=0.049$ ). The upper confidence interval and $P$-value were near the limit of nonsignificance. Furthermore, this association is not observed when tertile 2 is compared with tertile 1 (HR, 0.89; 95\% CI, 0.69-1.16). Similar conclusions were reached on yogurt intake, which was not significant when comparing tertile 2 with tertile 1 (HR, 0.84 ; 95\% CI, 0.64-1.10); the results were not well-stated. ${ }^{17)}$ In another prospective study conducted in China, the authors concluded that dairy consumption was associated with significantly lower risk of T2D. However, multivariable RRs for the fully adjusted model (i.e., model 4) inferred nonsignificant results with all dairy products considered in the study when comparing the highest intake quartiles with the lowest quartiles i.e., total dairy products (RR, 0.81 ; 95\% CI, 0.63-1.05; $P=0.07$ ), milk (RR, 0.92; 95\% CI, 0.77-1.12; $P=0.45)$, and total dairy without milk (RR, $0.77 ; 95 \% \mathrm{CI}, 0.58-1.03 ; P=0.11$ ). These comparisons were not considered in the study conclusions. ${ }^{18)}$

In the nested case-control Tehran Lipid and Glucose Study, the authors concluded a $27 \%$ significantly lower risk of T2D per $100 \mathrm{~g} /$ day total dairy consumption. This was actually nonsignificant with an odds ratio (OR) of 0.73 (95\% CI, $0.52-$ 1.02 ), and no significant findings were observed between any of the tertiles. Moreover, crude analysis revealed that men who consume cheese had $94 \%$ higher odds of T2D by comparing the highest tertile with the lowest tertile $(1.94 ; 95 \% \mathrm{CI}, 1.0-3.75){ }^{19)}$ In a cross-sectional study conducted in the Netherlands, the authors reported inverse association between skim milk dairy, fermented dairy, and buttermilk and onset of prediabetes; however, these results were actually nonsignificant $(\mathrm{OR}, 0.98$; 95\% CI, 0.97-1.00; OR, 0.98; 95\% CI, 0.97-0.99; and OR, 0.97; 95\% CI, 0.94-1.00; respectively). ${ }^{20)}$ In a population-based Singapore Chinese Health Study, the authors reported that dairy intake was significantly associated with reduced T2D risk when all other quartiles were compared with the lowest quartile. However, their HRs at 95\% CI revealed that associations were nonsignificant for the second, third, and fourth quartiles of dairy intake, i.e., 0.98 (0.91-1.06), $0.96(0.89-1.03)$, and 0.90 (0.83-0.98), respectively. ${ }^{21)}$ In a recent EPIC-InterAct study with a nested case-control design, the authors concluded inverse association between cheese intake (HR, 0.88 ; 95\% CI, 0.76-1.02) and combined fermented dairy intake (HR, $0.88 ; 95 \% \mathrm{CI}$, $0.78-0.99)$ and incidence of T2D. These results are actually not statistically significant, which was not reflected in their conclusion. ${ }^{22)}$ 


\section{Possible reasons for disparities in results}

First, the majority of studies have collected data through questionnaire-based responses. This potentially affects results due to recall bias and self-reported information. ${ }^{23-29)}$ Second, variations in diagnosis cutoffs among studies might give rise to differences in incident cases of T2D. Third, very few studies considered prediabetes as an outcome in assessing its relationship with dairy consumption, which may underestimate the actual risk of T2D. ${ }^{20,23)}$ Although the food frequency questionnaires utilized in these studies are extensively validated, there is an inevitable chance for measurement errors in capturing accurate data related to dairy and other foods that might incorporate certain disparities into the results. ${ }^{30)}$ Also, because most of these studies were observational in design, causal inference was not possible. Residual confounding is possible and might account for some of the differences in findings among studies. ${ }^{26-31)}$ Importantly, the findings of each study have limited generalizability to other populations with different characteristics.

\section{Evidence from systematic reviews and meta-analysis}

In a systematic review and dose-response meta-analysis published in 2013, the authors reported and concluded that yogurt intake is inversely associated with risk of T2D. However, this result was not statistically significant, i.e., RR, 0.91; 95\% CI, $0.82-1.00$. Furthermore, the conclusions were only directed toward products that are inversely associated. This ignores certain products, such as full-fat dairy, total milk, full-fat milk, cream, and fermented dairy products, which are positively or not associated with risk of dairy intake on T2D. ${ }^{32)}$ In another systematic review and dose-response meta-analysis of observational studies, the authors reported that total dairy and low-fat dairy were inversely associated with risk of T2D (RR, $0.97 ; 95 \% \mathrm{CI}, 0.95-1.00 ; P=0.04$ and RR, 0.96 ; $95 \% \mathrm{CI}, 0.92-1.00$; $P=0.072$ ). However, the upper confidence intervals for both products cross the level of nonsignificance, and one of the probability values is not significant. ${ }^{33}$

\section{Heterogeneity in findings among studies}

Although a study by Chen et al. ${ }^{34)}$ in 2015 noted a significantly reduced risk of T2D with yogurt intake $(0.82 ; 0.70-0.96)$, there is substantial variation in heterogeneity across studies $\left(I^{2}=65.3 \%\right)$. In a similar study by Gijsbers et al., ${ }^{33)}$ considerable variation due to heterogeneity was observed with some dairy products and risk of T2D, including total dairy $\left(I^{2}=66 \%\right)$, low-fat dairy $\left(I^{2}=68 \%\right)$, yogurt $\left(I^{2}=73 \%\right)$, and ice cream $\left(I^{2}=86 \%\right){ }^{33)}$ In a recent meta-analysis, significant heterogeneity was observed in RR of low-fat dairy $\left(I^{2}=65.6 \%\right)$ and rate ratios for total dairy $\left(I^{2}=78 \%\right)$ and low-fat dairy $\left(I^{2}=78.3 \%\right)$, although they exhibited an inverse association. ${ }^{35)}$ Although low-fat dairy and yogurt have shown inverse relationships with risk of dairy on T2D, there is remarkable variation in dispersion of effect sizes, with
$I^{2}=94 \%$ and $I^{2}=70 \%$, respectively. ${ }^{36)}$ Lastly, findings from a dose-response meta-analysis by Gao et al. ${ }^{32)}$ inferred that total dairy $\left(I^{2}=65.4 \%\right)$, yogurt $\left(I^{2}=74 \%\right)$, and cheese $(I 2=59 \%)$ had an inverse association with T2D. However, these results also exhibit high heterogeneity across studies. ${ }^{32)}$

\section{Discussion}

Although most of the studies projected significant reduction in risk of T2D with dairy consumption, the above statement holds true only for certain food products such as low-fat dairy, particularly yogurt and cheese. Notably, other studies showed no association with low-fat dairy, ${ }^{16)}$ yogurt, and cheese intake, ${ }^{19)}$ on incidence of T2D. Through this review, the authors intend to demonstrate that not all dairy foods have a beneficial role in preventing T2D, as most dairy foods (butter, sour/whipped cream, ice creams, whole/skim milk, dairy desserts, unfermented products, and whole/full/high-fat dairy foods) showed not statistically significant findings. Conversely, a few studies have shown harmful effects of dairy, especially whole milk or unfermented milk, ${ }^{13)}$ low-fat dairy, ${ }^{16)}$ and cheese. ${ }^{19)}$

Even after extensive research investigating the link between dairy consumption and risk of T2D, there is a wide range of mixed findings resulting in significant uncertainty. Additional studies exploring confounding factors such as race, geographic localization, ethnicity, dietary patterns, food intake behavior, and lifestyle factors are needed to ascertain the potential detrimental effects that may have had a role in contributing to these disparate conclusions. The public should consider multiple factors, including study generalizability, when drawing conclusions about the association of dairy and T2D. Decisions should not rely only on study conclusions, as most studies focus on the positive outcomes and ignore neutral or negative findings. We suggest that both favorable and unfavorable findings should be reported to allow readers and the public to make informed decisions. Researchers should consider both statistical and clinical significance and prioritize confidence intervals rather than $P$-values when interpreting study findings. Study results should be appropriately interpreted to prevent under- and overreporting, and conclusions should correspond with results. After considering all available evidence, dairy products as part of a healthy diet plan do not show harmful effects on glycemic control. However, the potential benefits of dairy consumption on plasma glucose level, insulin sensitivity, and other T2D-related outcomes warrant future investigations with long-term, well-designed clinical trials.

In conclusion, not all dairy products exhibit a beneficial role in preventing T2D. Low-fat dairy foods, especially yogurt, do have a beneficial role, while most other dairy foods show no association. Readers and the public should maintain caution when assessing new studies and consider heterogeneity, generalizability, and statistical and clinical significance when making decisions. 


\section{Conflicts of interest}

No potential conflict of interest relevant to this article was reported.

\section{References}

1. International Diabetes Federation. IDF Diabetes Atlas. 9th edition [Internet]. Brussels (Belgium): International Diabetes Federation, 2019 [cited 2020 Feb 15]. Available from: https://www.diabetesatlas.org.

2. The Global Diabetes Community. Diabetes prevalence [Internet]. Diabetes.co.uk - the global diabetes community; 2019 [cited 2020 Feb 15]. Available from: https://www. diabetes.co.uk/diabetes-prevalence.html.

3. American Diabetes Association. Diabetes risk [Internet]. Arlington (VA): American Diabetes Association, 2019 [cited 2020 Feb 15]. Available from: https://www.diabetes. org/diabetes-risk.

4. Canadian Diabetes Association. Signs, risks \& prevention [Internet]. Toronto (ON): Canadian Diabetes Association; 2019 [cited 2020 Feb 15]. Available from: https://www. diabetes.ca/signs,-risks---prevention/risk-factors--assessments.

5. Gudi SK. Eating speed and the risk of type 2 diabetes: explorations based on real-world evidence. Ann Pediatr Endocrinol Metab 2020;25:80-3.

6. Gil Á, Ortega RM. Introduction and executive summary of the supplement, role of milk and dairy products in health and prevention of noncommunicable chronic diseases: a series of systematic reviews. Adv Nutr 2019;10 (suppl_2):S67-73.

7. Rozenberg S, Body JJ, Bruyère O, Bergmann P, Brandi ML, Cooper C, et al. Effects of dairy products consumption on health: benefits and beliefs--a commentary from the belgian bone club and the European Society for Clinical and Economic Aspects of Osteoporosis, Osteoarthritis and Musculoskeletal Diseases. Calcif Tissue Int 2016;98:1-17.

8. Guasch-Ferré M, Becerra-Tomás N, Ruiz-Canela M, Corella D, Schröder H, Estruch R, et al. Total and subtypes of dietary fat intake and risk of type 2 diabetes mellitus in the Prevención con Dieta Mediterránea (PREDIMED) study. Am J Clin Nutr 2017;105:723-35.

9. Trichia E, Luben R, Khaw KT, Wareham NJ, Imamura F, Forouhi NG. The associations of longitudinal changes in consumption of total and types of dairy products and markers of metabolic risk and adiposity: findings from the European Investigation into Cancer and Nutrition (EPIC)-Norfolk study, United Kingdom. Am J Clin Nutr 2020;111:1018-26.

10. Abreu S, Moreira P, Moreira C, Mota J, Moreira-Silva I, Santos PC, et al. Intake of milk, but not total dairy, yogurt, or cheese, is negatively associated with the clustering of cardiometabolic risk factors in adolescents. Nutr Res 2014;34:48-57.
11. Lu W, Chen H, Niu Y, Wu H, Xia D, Wu Y. Dairy products intake and cancer mortality risk: a meta-analysis of 11 population-based cohort studies. Nutr J 2016;15:91.

12. Malekinejad H, Rezabakhsh A. Hormones in dairy foods and their impact on public health - a narrative review article. Iran J Public Health 2015;44:742-58.

13. Choi HK, Willett WC, Stampfer MJ, Rimm E, Hu FB. Dairy consumption and risk of type 2 diabetes mellitus in men: a prospective study. Arch Intern Med 2005;165:997-1003.

14. Liu S, Choi HK, Ford E, Song Y, Klevak A, Buring JE, et al. A prospective study of dairy intake and the risk of type 2 diabetes in women. Diabetes Care 2006;29:1579-84.

15. Kirii K, Mizoue T, Iso H, Takahashi Y, Kato M, Inoue M, et al. Calcium, vitamin D and dairy intake in relation to type 2 diabetes risk in a Japanese cohort. Diabetologia 2009;52:2542-50.

16. Louie JC, Flood VM, Rangan AM, Burlutsky G, Gill TP, Gopinath B, et al. Higher regular fat dairy consumption is associated with lower incidence of metabolic syndrome but not type 2 diabetes. Nutr Metab Cardiovasc Dis 2013;23:816-21.

17. O'Connor LM, Lentjes MA, Luben RN, Khaw KT, Wareham NJ, Forouhi NG. Dietary dairy product intake and incident type 2 diabetes: a prospective study using dietary data from a 7-day food diary. Diabetologia 2014;57:909-17.

18. Zong G, Sun Q, Yu D, Zhu J, Sun L, Ye X, et al. Dairy consumption, type 2 diabetes, and changes in cardiometabolic traits: a prospective cohort study of middle-aged and older Chinese in Beijing and Shanghai. Diabetes Care 2014;37:56-63.

19. Moslehi N, Shab-Bidar S, Mirmiran P, Sadeghi M, Azizi F. Associations between dairy products consumption and risk of type 2 diabetes: Tehran lipid and glucose study. Int J Food Sci Nutr 2015;66:692-9.

20. Brouwer-Brolsma EM, Sluik D, Singh-Povel CM, Feskens EJM. Dairy product consumption is associated with prediabetes and newly diagnosed type 2 diabetes in the Lifelines Cohort Study. Br J Nutr 2018;119:442-55.

21. Talaei M, Pan A, Yuan JM, Koh WP. Dairy intake and risk of type 2 diabetes. Clin Nutr 2018;37:712-8.

22. Sluijs I, Forouhi NG, Beulens JW, van der Schouw YT, Agnoli C, Arriola L, et al. The amount and type of dairy product intake and incident type 2 diabetes: results from the EPIC-InterAct Study. Am J Clin Nutr 2012;96:382-90.

23. Hruby A, Ma J, Rogers G, Meigs JB, Jacques PF. Associations of dairy intake with incident prediabetes or diabetes in middle-aged adults vary by both dairy type and glycemic status. J Nutr 2017;147:1764-75.

24. Grantham NM, Magliano DJ, Hodge A, Jowett J, Meikle P, Shaw JE. The association between dairy food intake and the incidence of diabetes in Australia: the Australian Diabetes Obesity and Lifestyle Study (AusDiab). Public Health Nutr 2013;16:339-45.

25. Jeon J, Jang J, Park K. Effects of consuming calcium-rich foods on the incidence of type 2 diabetes mellitus. Nutrients $2018 ; 11: 31$. 
26. Eussen SJ, van Dongen MC, Wijckmans N, den Biggelaar L, Oude Elferink SJ, Singh-Povel CM, et al. Consumption of dairy foods in relation to impaired glucose metabolism and type 2 diabetes mellitus: the Maastricht Study. Br J Nutr 2016;115:1453-61.

27. Ardisson Korat AV, Li Y, Sacks F, Rosner B, Willett WC, $\mathrm{Hu} \mathrm{FB}$, et al. Dairy fat intake and risk of type 2 diabetes in 3 cohorts of US men and women. Am J Clin Nutr 2019;110:1192-200.

28. Drouin-Chartier JP, Li Y, Ardisson Korat AV, Ding M, Lamarche B, Manson JE, et al. Changes in dairy product consumption and risk of type 2 diabetes: results from 3 large prospective cohorts of US men and women. Am J Clin Nutr 2019;110:1201-12.

29. Struijk EA, Heraclides A, Witte DR, Soedamah-Muthu SS, Geleijnse JM, Toft U, et al. Dairy product intake in relation to glucose regulation indices and risk of type 2 diabetes. Nutr Metab Cardiovasc Dis 2013;23:822-8.

30. Drehmer M, Pereira MA, Schmidt MI, Del Carmen B Molina M, Alvim S, Lotufo PA, et al. Associations of dairy intake with glycemia and insulinemia, independent of obesity, in Brazilian adults: the Brazilian Longitudinal Study of Adult Health (ELSA-Brasil). Am J Clin Nutr 2015;101:775-82.
31. Lago-Sampedro A, García-Escobar E, Rubio-Martín E, Pascual-Aguirre N, Valdés S, Soriguer F, et al. Dairy product consumption and metabolic diseases in the Di@bet.es study. Nutrients 2019;11:262.

32. Gao D, Ning N, Wang C, Wang Y, Li Q, Meng Z, et al. Dairy products consumption and risk of type 2 diabetes: systematic review and dose-response meta-analysis. PLoS One 2013;8:e73965.

33. Gijsbers L, Ding EL, Malik VS, de Goede J, Geleijnse JM, Soedamah-Muthu SS. Consumption of dairy foods and diabetes incidence: a dose-response meta-analysis of observational studies. Am J Clin Nutr 2016;103:1111-24.

34. Chen M, Sun Q, Giovannucci E, Mozaffarian D, Manson JE, Willett WC, et al. Dairy consumption and risk of type 2 diabetes: 3 cohorts of US adults and an updated metaanalysis. BMC Med 2014;12:215.

35. Khoramdad M, Alimohamadi Y, Safiri S, Pakzad R, Shakiba E, Shafiei J, et al. Dairy products consumption and risk of type 2 diabetes: a systematic review and meta-analysis / of prospective cohort studies. Iran Red Crescent Med J 2017;19:e14140.

36. Aune D, Norat T, Romundstad P, Vatten LJ. Dairy products and the risk of type 2 diabetes: a systematic review and dose-response meta-analysis of cohort studies. Am J Clin Nutr 2013;98:1066-83. 